\title{
PRESERVATION OF $p$-POINCARÉ INEQUALITY FOR LARGE $p$ UNDER SPHERICALIZATION AND FLATTENING
}

\author{
ESTIBALITZ DURAND-CARTAGENA AND XINING LI
}

\begin{abstract}
Li and Shanmugalingam showed that annularly quasiconvex metric spaces endowed with a doubling measure preserve the property of supporting a $p$-Poincaré inequality under the sphericalization and flattening procedures. Because natural examples such as the real line or a broad class of metric trees are not annularly quasiconvex, our aim in the present paper is to study, under weaker hypotheses on the metric space, the preservation of $p$-Poincaré inequalites under those conformal deformations for sufficiently large $p$. We propose the hypotheses used in a previous paper by the same authors, where the preservation of $\infty$-Poincaré inequality has been studied under the assumption of radially star-like quasiconvexity (for sphericalization) and meridian-like quasiconvexity (for flattening). To finish, using the sphericalization procedure, we exhibit an example of a Cheeger differentiability space whose blow up at a particular point is not a PI space.
\end{abstract}

\section{Introduction}

One of the cornerstones in the development of first order calculus in the metric measure setting is the concept of metric space equipped with a doubling measure and supporting a Poincaré inequality. If a space or domain supports

Received April 25, 2016; received in final form October 10, 2016.

The research of the first author was partially supported by the grant MTM2012-34341 (Spain) and the research of the second author was partially supported by the NSF grant DMS-1200915. This research began during the visit of the first author to the Department of Mathematical Sciences at University of Cincinnati in the Spring 2014 and continue during the visit of the second author (supported by 2016-MAT09) to the Departamento de Matemática Aplicada, ETSI Industriales (UNED) in the Spring 2016. The authors would like to thank these institutions for its kind hospitality.

2010 Mathematics Subject Classification. Primary 31E05. Secondary 30L10, 30L99. 
a Poincaré inequality, many fruitful geometric and analytical properties can be deduced, including the existence of non-trivial differentiable structures. Therefore, it is valuable to explore which metric spaces enjoy such properties. For a general introduction to the subject one can look at [2], [13], [15] or [16].

A common way to construct new metric spaces from old ones is to use conformal deformations in the sense of [1]. This means to construct a new metric space, which is homeomorphic to the original one, by endowing the old space with a new metric density function. In order to preserve certain geometric properties, the measure also plays an important role and should be altered in a similar way. A natural problem is to study the preservation of the doubling property and the Poincaré inequality under these deformations. In the present paper, two types of conformal deformations are considered: sphericalization and flattening.

Sphericalization and flattening are dual transformations in the sense that if one starts from a bounded metric space, then performs a flattening transformation followed by a sphericalization transformation, then the resulting metric space is biLipschitz equivalent to the original space. Furthermore, starting from an unbounded metric space, the performance of sphericalization followed by a flattening transformation leads to a metric space that is biLipschitz equivalent to the original.

The idea of sphericalization and flattening was first considered by Bonk and Kleiner [5] (sphericalization) and Balogh and Buckley [1] and further studied in [6] and [17]. Within these papers, two types of conformal deformations were introduced in order to generalize the stereographic projection between the Riemann sphere and the complex plane. Their motivation comes from comparing quasihyperbolic metrics of a domain (which are considered in Bonk, Heinonen and Koskela [4]) with two types of metric, the length metric and the sphericalized metric on the domain.

The preservation of $p$-Poincaré inequality under these conformal deformations for the case $p<\infty$ was first studied in Li and Shanmugalingam [20], assuming that the original space is annularly quasiconvex. By a result in Korte [19], spaces supporting a $p$-Poincaré inequality for sufficiently small $p \geq 1$ are necessarily annularly quasiconvex. It was shown in [20] that the property of annular quasiconvexity cannot be removed in their results regarding preservation of the property of supporting a $p$-Poincaré inequality for sufficiently small $p$, and the authors of [20] pose whether the assumption of annular quasiconvexity is necessary for preserving a $p$-Poincaré inequality for sufficiently large $p$.

At this point, it is important to highlight the role that the exponent $p$ plays in $p$-Poincaré inequalities. The larger the $p$, the weaker the inequality and the limiting case, the $\infty$-Poincaré inequality, would be the weakest. We refer the interested reader to [21] or [12] for several examples of spaces supporting a $p$-Poincaré inequality for some but not all values of $p$ in the range $[1, \infty]$. 
In [9], the preservation of quasiconvexity and $\infty$-Poincaré inequality has been studied under a weaker assumption, namely, radially star-like quasiconvexity and meridian-like quasiconvexity. The motivation for introducing these new definitions comes from the fact that there are simple examples that are not annularly quasiconvex but still support a $p$-Poincaré inequality, for example the real line $\mathbb{R}$ or $\mathbb{S}^{1}$ when endowed with the length metric. Actually, the sphericalization of $\mathbb{R}$ gives $\mathbb{S}^{1}$ and viceversa. The definition of such properties is inspired by the paper [4], where the authors considered the duality of uniform domains and Gromov hyperbolic spaces and use the concept of rough star-likeness.

The different nature of $p$-Poincaré inequality for finite $p$ versus $\infty$-Poincaré inequality makes that the techniques used in [20] differ from the ones used in [9]. In [20], a version of Boman type chaining arguments found in [3] and [12] is used. In [9], the authors consider the case $p=\infty$ and use a purely geometric characterization of $\infty$-Poincaré inequality proved in [10] and based on a stronger version of quasiconvexity.

For a metric space supporting a doubling measure there are two exponents related to the doubling measure, the relative upper bound exponent $t$ and the relative lower bound exponent $s$ with $t \leq s$ in general. In the present paper, we improve part of the results in [20], namely, the preservation of $p$-Poincaré inequality under sphericalization and flattening for $p>s$, under assumptions that are weaker than annulular quasiconvexity: radially star-like quasiconvexity for sphericalization, and meridian-like quasiconvexity for flattening. On the other hand, it is well-known that Ahlfors $Q$-regular spaces that support a a $p$-Poincaré inequality for some $1 \leq p<Q$ are annularly quasiconvex when $Q>1$ (see [19]). Notice that in this case $t=s=Q$ and therefore, Ahlfors $Q$ regular spaces with $Q>1$ preserve the $p$-Poincaré inequality for $p>1$ under sphericalization and flattening procedures.

It is an open question (see for example [8]) whether the blow-up of a positive measure subset of a differentiability space (in the sense of Cheeger) must be a PI space, that is, a metric space with a doubling measure and a $p$-Poincaré inequality for some $p<\infty$. Using the sphericalization procedure, we make a first step in this direction by exhibiting in Example 2 a differentiability space whose blow up at a particular point is not a PI space.

The key point of this construction is that, as opposed to the examples that appear in [11] (in which the $p$-Poincaré inequality only fails at large scales), the $p$-Poincaré inequality fails for domains that contain the image of infinity from the original space.

The paper is organized as follows: in Section 2, basic notations and definitions are introduced; in Section 3, preservation of $p$-Poincaré inequality for $p>s$ under sphericalization for radially star-like quasiconvex spaces is proved (see Theorem 3.1). In Section 4, preservation of $p$-Poincaré inequality for $p>s$ 
under flattening for meridian-like quasiconvex spaces is presented (see Theorem 4.1). Last section, Section 5, contains an example of a differentiability space whose blow-up is not a PI space.

\section{Notation and preliminaries}

In this section, we gather the key notions, definitions and notations that will be used throughout the paper.

2.1. Curves in metric spaces. Let $(X, d)$ be a metric space. We denote open balls centered at $x \in X$ and of radius $r>0$ by $B(x, r):=\{y \in$ $X: d(x, y)<r\}$ and closed balls by $\bar{B}(x, r):=\{y \in X: d(x, y) \leq r\}$. For $\lambda>0$, $\lambda B$ denotes the ball concentric with $B$ (with respect to a predetermined center) but with radius $\lambda$-times the radius of $B$. For $0<r<R, A(a, r, R)$ denotes the annulus $A(a, r, R):=\bar{B}(a, R) \backslash B(a, r)$.

Given a continuous map (also known as curve) $\gamma: I \rightarrow X$, where $I=[a, b]$ for some $a, b \in \mathbb{R}$ with $a<b$, we denote the length of $\gamma$ with respect to the metric $d$ by

$$
\ell_{d}(\gamma):=\sup \sum_{k=0}^{n-1} d\left(\gamma\left(t_{k}\right), \gamma\left(t_{k+1}\right)\right),
$$

where the supremum is taken over all partitions $a=t_{0}<t_{1}<\cdots<t_{n}=b$ of the interval $[a, b]$. A curve $\gamma$ is rectifiable if $\ell_{d}(\gamma)<\infty$. We simply write $\ell(\gamma)$ if the metric is clear from the context. Given two points $x, y \in X, \gamma_{x y}$ denotes a curve connecting $x$ to $y$.

For a rectifiable curve $\gamma:[a, b] \rightarrow X$, let $s_{\gamma}:[a, b] \rightarrow[0, \ell(\gamma)]$ be the associated length function. That is, $s_{\gamma}(t)=\ell\left(\gamma_{\mid[a, t]}\right)$. There exists a unique (1Lipschitz continuous) map $\gamma_{s}:[0, \ell(\gamma)] \rightarrow X$ such that $\gamma=\gamma_{s} \circ s_{\gamma}$. The curve $\gamma_{s}$ is called the arc length parametrization of $\gamma$. The image of a curve will be denoted by $|\gamma|$. If $\gamma$ is a rectifiable curve in $X$, the line integral over $\gamma$ of a Borel function $\rho: X \rightarrow[0, \infty]$ is defined by

$$
\int_{\gamma} \rho d s:=\int_{0}^{\ell(\gamma)}\left(\rho \circ \gamma_{s}\right)(t) d t .
$$

A metric space $(X, d)$ is said to be $C$-quasiconvex if there exists $C \geq 1$ such that for every pair of points $x$ and $y$ there exists a rectifiable curve $\gamma$ with $\ell_{d}(\gamma) \leq C d(x, y)$. A related notion to quasiconvexity is that of annular quasiconvexity, a notion introduced in [19] that has been further used in [6], [13] and [17], for example. We say that $X$ is A-annularly quasiconvex with respect to a base point $a \in X$ if there exists $A \geq 1$ such that for every $r>0$, and for each pair of points $x, y \in A(a, r / 2, r)$ there is a curve $\gamma_{x y}$ connecting $x$ to $y$ inside the annulus $A(a, r / A, A r)$ with $\ell_{d}(\gamma) \leq A d(x, y)$. We say that $(X, d)$ is annularly quasiconvex if there exists $A \geq 1$ such that $X$ is $A$-annularly quasiconvex for every $a \in X$. 
2.2. Metric measure spaces. A metric space $(X, d)$ endowed with a Borel measure $\mu$ is called a metric measure space and will be denoted by $(X, d, \mu)$. We say that the measure $\mu$ is doubling if balls have finite positive measure and there is a constant $C_{\mu} \geq 1$ such that

$$
\mu(2 B) \leq C_{\mu} \mu(B)
$$

for all balls $B$ in $X$.

A metric space is called doubling if there is a constant $C$ so that every ball of radius $r$ can be covered by at most $C$ balls of radius $r / 2$. It is well known that a complete metric space $X$ admits a doubling measure if and only if $X$ is doubling. Moreover, a complete doubling metric space is proper, that is, every closed ball in it is compact. See [16, Section 4].

Condition (1) implies that there are constants $C>0$ and $s>0$, depending only on $C_{\mu}$, such that

$$
\frac{\mu(B(x, r))}{\mu(B(y, R))} \geq C\left(\frac{r}{R}\right)^{s}
$$

whenever $0<r \leq R$ and $x \in B(y, R)$. See [13] for a proof of this fact. In this case, we also say that $X$ has a relative lower volume decay of order $s>0$.

If the measure is doubling and the space is connected, then there exist an exponent $t>0$ and a constant $C>0$ such that

$$
\frac{\mu(B(x, r))}{\mu(B(y, R))} \leq C\left(\frac{r}{R}\right)^{t}
$$

for $0<r \leq R \leq \operatorname{diam} X / 2$ and $x \in B(y, R)$. In general, we have $s \geq t$, and we say that $X$ has a relative upper volume decay of order $t>0$.

2.3. First-order calculus in metric measure spaces. Given a real-valued function $u$ in a metric space $X$, a Borel function $g: X \rightarrow[0, \infty]$ is an upper gradient of $u$ if

$$
|u(x)-u(y)| \leq \int_{\gamma} g d s,
$$

for each rectifiable curve $\gamma$ connecting $x$ to $y$ in $X$.

Given $1 \leq p<\infty$, we say that $(X, d, \mu)$ supports a $p$-Poincaré inequality if each ball in $X$ has finite and positive measure and there are constants $C, \lambda>0$ such that for every open ball $B$ in $X$, for every measurable function $u$ on $B$, and for every upper gradient $g$ of $u$ we have

$$
\frac{1}{\mu(B)} \int_{B}\left|u-u_{B}\right| d \mu \leq C \operatorname{rad}(\lambda B)\left[\left(\frac{1}{\mu(\lambda B)} \int_{\lambda B} g^{p} d \mu\right)^{1 / p}\right] .
$$

Here $\operatorname{rad}(\lambda B)$ denotes the radius of the ball $\lambda B$, and for arbitrary $A \subset X$ with $0<\mu(A)<\infty$ we write $u_{A}=\frac{1}{\mu(A)} \int_{A} u d \mu=f_{A} u d \mu$.

When $p=\infty$, the term inside the square brackets of the above inequality should be interpreted to mean $\|g\|_{L^{\infty}(\lambda B)}$. 
The following result due to Keith [18] states that to verify a $p$-Poincaré inequality it suffices to verify the inequality for Lipschitz functions and their continuous upper gradients.

Lemma 2.1 ([18, Theorem 2]). Let $p \geq 1$ and let $(X, d, \mu)$ be a complete metric measure space with $\mu$ doubling. Then the following conditions are quantitatively equivalent:

(a) $(X, d, \mu)$ admits a p-Poincaré inequality for all measurable functions and their upper gradients.

(b) $(X, d, \mu)$ admits a p-Poincaré inequality for all compactly supported Lipschitz functions and their compactly supported Lipschitz upper gradients.

By the work of Cheeger [7], metric measure spaces endowed with a doubling measure and supporting a $p$-Poincaré inequality for $p<\infty$ have a very rich infinitesimal "linear" structure that allows to state the Rademacher differentiability theorem in this context. It is worth mentioning that a complete metric space supporting a doubling measure and a $p$-Poincaré inequality is quasiconvex (see [7, Theorem 17.1]) as defined in Section 2.1.

The interested reader can find in [16] a discussion of the recent advances in the field of analysis on metric measure spaces, including those in [20] (see also [16, Chapter 14]).

2.4. Sphericalization and flattening. The concept of sphericalization and flattening are natural analogs of the stereographic projection between the Riemann sphere and the complex plane. As pointed out in the Introduction, these notions were introduced by Bonk and Kleiner in [5] (sphericalization) and by Balogh and Buckley in [1] and further studied in [6] and [17].

For an unbounded locally compact metric space $X$, we denote its one-point compactification by $\dot{X}:=X \cup\{\infty\}$, where $U$ is open in $\dot{X}$ if either $U$ is open in $X$ or $U$ contains $\infty$ and $X \backslash U$ is a compact subset of $X$. In particular, $\dot{X}$ is compact with this topology.

Definition 2.2 (Sphericalization). Given a complete unbounded metric space $(X, d)$ and a base point $a \in X$, we consider the following density function $d_{a}: \dot{X} \times \dot{X} \rightarrow[0, \infty)$ given by

$$
d_{a}(x, y)= \begin{cases}\left.\frac{d(x, y)}{[1+d(x, a)][1+d(y, a)]}\right] & \text { if } x, y \in X \\ \frac{1}{1+d(x, a)} & \text { if } x \in X, y=\infty \\ 0 & \text { if } x=\infty=y\end{cases}
$$

Although $d_{a}$ is not a metric since it is possible to violate the triangular inequality, there exists a metric $\hat{d}_{a}$ on $\dot{X}$ whose metric topology agrees with the topology of $\dot{X}$ and satisfying

$$
\frac{1}{4} d_{a}(x, y) \leq \hat{d}_{a}(x, y) \leq d_{a}(x, y)
$$


for all $x, y \in \dot{X}$ (see [5, Lemma 2.2]).

The metric space $\left(\dot{X}, \hat{d}_{a}\right)$ is said to be the sphericalization of $(X, d)$. As shown in [1], the metric space resulting from flattening the (bounded) sphericalized space $\left(\dot{X}, \hat{d}_{a}\right)$ with respect to the point $\{\infty\}$ is bi-Lipschitz equivalent to the (unbounded) space $(X, d)$, making sphericalization and flattening dual transformations.

Since there is no closed form formula for $\hat{d}_{a}$, for convenience we will use $d_{a}$ in defining balls in $\dot{X}$. Furthermore, observe that $\operatorname{diam}(\dot{X})=1$. Balls in $\dot{X}$, with respect to $d_{a}$, will be denoted $B_{a}=B_{a}(x, r)$, while the balls in $X$, with respect to the original metric $d$, will be denoted $B=B(x, r)$. In a similar fashion, for $0<r<R$, an annulus with respect to the metric $d_{a}$ will be denoted by $A_{a}(a, r, R)$. Notice that the density function $d_{a}$ used here satisfies the condition of the standard sphericalizing function $g(t)=(1+t)^{-2}$ as in $[1$, Section 2]. Actually, because $g$ is continuous on $X$, it can be proved that the length metric (associated with $g$ ) as considered in [1, Section 2] is biLipschitz equivalent to the metric $\hat{d}_{a}$, with biLipschitz constant only depending on the quasiconvexity constant of $X$.

The operation of flattening, which is dual to the procedure of sphericalization, can be defined analogously. In the flattening procedure, we begin with a bounded metric space and remove a point to construct an unbounded metric space.

Definition 2.3 (Flattening). Given a complete bounded metric space $(X, d)$ with a base point $c \in X$, we consider the metric space $X^{c}:=X \backslash\{c\}$, with a density function $d^{c}: X^{c} \times X^{c} \rightarrow[0, \infty)$ defined by

$$
d^{c}(x, y)=\frac{d(x, y)}{d(x, c) d(y, c)} \quad \text { if } x, y \in X^{c} .
$$

Just as in the case of sphericalization, the density function $d^{c}$ is not a metric, but by $\left[6\right.$, Lemma 3.2], we have a metric space $\left(X^{c}, \bar{d}\right)$ associated to $d^{c}$ with

for all $x, y \in X^{c}$.

$$
\frac{1}{4} d^{c}(x, y) \leq \bar{d}(x, y) \leq d^{c}(x, y)
$$

The metric space $\left(X^{c}, \bar{d}\right)$ is said to be a flattening of $(X, d)$. Balls in $X^{c}$, with respect to the metric $d^{c}$, will be denoted $B^{c}(x, r)$. An annulus centered at $x$ with respect to the metric $d^{c}$ will be denoted by $A^{c}(x, r, R)$, where $0<r<R$.

In the sequel, it will be useful to know how a curve and its corresponding length change under the sphericalization and flattening processes. Let $\gamma$ be a rectifiable curve in a rectifiably connected unbounded metric space $X$. Under sphericalization $\gamma$ corresponds to $\dot{\gamma}:[0, \ell(\gamma)] \rightarrow \dot{X}$ defined by $\dot{\gamma}(t)=\gamma_{s}(t)$, where $\gamma_{s}$ is the arc-length parametrization of $\gamma$ with respect to the original metric $d$. By an abuse of notation, we will denote the corresponding curve in $\dot{X}$ by $\gamma$ as well. One can check (see [1, Proposition 2.6]) that $\gamma$ is rectifiable 
with respect to the metric $\widehat{d}_{a}$ if it is rectifiable with respect to the original metric $d$.

Then length $\ell_{d_{a}}(\gamma)$ of $\gamma$ with respect to "the metric" $d_{a}$ is is given by the formula

$$
\ell_{d_{a}}(\gamma)=\int_{0}^{\ell(\gamma)} \frac{1}{\left[1+d\left(\gamma_{s}(t), a\right)\right]^{2}} d s(t)
$$

whereas the formula for the length $\ell_{d^{c}}(\gamma)$ of $\gamma$ with respect to "the metric" $d^{c}$ is given by

$$
\ell_{d^{c}}(\gamma)=\int_{0}^{\ell(\gamma)} \frac{1}{d\left(\gamma_{s}(t), c\right)^{2}} d s(t) .
$$

In the next lemma, we explain how upper gradients are transformed under sphericalization. Note that a function that is Lipschitz continuous on $X$ will be locally Lipschitz continuous on $\dot{X} \backslash\{\infty\}$, and a function that is Lipschitz continuous on $\dot{X}$ is necessarily Lipschitz continuous on $X$.

LEMMA 2.4 ([20, Lemma 3.3.1]). Suppose that $u$ is a Lipschitz function on $\dot{X}$. If $g$ is an upper gradient of $u$ in $X$, then the function $\hat{g}$ given by

$$
\hat{g}(x)=g(x)(1+d(x, a))^{2}
$$

and extended by setting $\hat{g}(\infty)=0$ is an upper gradient of $u$ in $\dot{X}$. Furthermore, if $h$ is an upper gradient of a function $v$ in $\dot{X}$, then the function $\bar{h}$ given by

$$
\bar{h}(x)=\frac{h(x)}{(1+d(x, a))^{2}}
$$

is an upper gradient of $v$ in $X$.

The current work focuses on the preservation of Poincaré inequalities in the setting of metric measure spaces under sphericalization and flattening, so we also need to transform the measure on $X$ in a manner compatible with the change in the metric.

Definition 2.5. Suppose $(X, d)$ is proper space equipped with a Borelregular measure $\mu$ such that the measures of non-empty open bounded sets are positive and finite. We consider the spherical measure $\mu_{a}$ defined on $\dot{X}$ as follows. For a Borel set $A \subset \dot{X}$, the measure $\mu_{a}(A)$ is given by

$$
\mu_{a}(A)=\int_{A \backslash\{\infty\}} \frac{1}{\mu(B(a, 1+d(z, a)))^{2}} d \mu(z) .
$$

We next define the transformation $\mu^{c}$ of the measure $\mu$ under flattening. In this case, $X$ is a bounded metric space equipped with a Borel-regular measure $\mu$. 
Definition 2.6. The flattened measure $\mu^{c}$ corresponding to $\left(X^{c}, d^{c}\right)$ is given by

$$
\mu^{c}(A)=\int_{A} \frac{1}{\mu(B(c, d(c, z)))^{2}} d \mu(z),
$$

whenever $A \subset X^{c}$ is a Borel set.

The spherical and the flattened measure are doubling if the original measure $\mu$ is doubling as well. See [20].

The following lemma, due to N. Shanmugalingam, shows the corresponding result for measures. We are grateful to N. Shanmugalingam for allowing us to include the result here.

LEMMA 2.7. Let $X$ be a connected, unbounded, complete metric measure space and $\mu$ be a doubling measure on $X$. Let $a \in X$ and $X_{a}=X \cup\{\infty\}$ be the sphericalization of $X$ with respect to the base point $a$, and $X_{a}^{\infty}$ be the flattening of $X_{a}$ with respect to the base point $\infty$. Then $\mu_{a}^{\infty} \approx \mu$, that is, there is a constant $C>0$ such that for all $x \in X$,

$$
\frac{1}{C} d \mu(x) \leq d \mu_{a}^{\infty}(x) \leq C d \mu(x)
$$

and $\mu, \mu_{a}^{\infty}$ are mutually absolutely continuous.

Proof. The fact that $\mu$ and $\mu_{a}^{\infty}$ are absolutely continuous with respect to each other is clear from the definitions of $\mu_{a}$ and $\mu_{a}^{\infty}$.

Note that

$$
\begin{aligned}
d \mu_{a}^{\infty}(x) & =\frac{d \mu_{a}(x)}{\mu_{a}\left(B_{a}\left(\infty, d_{a}(x, \infty)\right)\right)^{2}} \\
& =\frac{d \mu(x)}{\mu_{a}\left(B_{a}\left(\infty, d_{a}(x, \infty)\right)\right)^{2} \mu(B(a, 1+d(x, a)))^{2}}
\end{aligned}
$$

Thus, we consider $\mu_{a}\left(B_{a}\left(\infty, d_{a}(x, \infty)\right)\right)$. Observe that $y \in B_{a}\left(\infty, d_{a}(x, \infty)\right)$ if and only if $d_{a}(y, \infty)<d_{a}(x, \infty)$, that is, $d(y, a)>d(x, a)$. It follows that

$$
B_{a}\left(\infty, d_{a}(x, \infty)\right)=X \backslash \bar{B}(a, d(x, a)) .
$$

The rest of the proof is divided into two cases.

Case 1: $d(x, a)>1 / C$. In this case, note that for $y \in X \backslash B(a, d(x, a))$ we have that $1+d(y, a) \approx d(y, a)$ and hence by the doubling property of $\mu$, we also have $\mu(B(a, 1+d(y, a))) \approx \mu(B(a, d(y, a)))$. For non-negative integers $j$ we set $B_{j}=B\left(a, 2^{j} d(x, a)\right)$. Then by the doubling property of $\mu$,

$$
\begin{aligned}
\mu_{a}\left(B_{a}\left(\infty, d_{a}(\infty, x)\right)\right) & \approx \int_{X \backslash \bar{B}(a, d(x, a))} \frac{1}{\mu(B(a, d(y, a)))^{2}} d \mu(y) \\
& =\sum_{j=0}^{\infty} \int_{B_{j+1} \backslash \bar{B}_{j}} \frac{1}{\mu(B(a, d(y, a)))^{2}} d \mu(y)
\end{aligned}
$$




$$
\approx \sum_{j=0}^{\infty} \frac{\mu\left(B_{j+1} \backslash \bar{B}_{j}\right)}{\mu\left(B_{j}\right)^{2}}
$$

By (2) and (3), there are positive constants $t, s$ (which are independent of $j, x)$ such that $\mu\left(B_{j+1} \backslash \bar{B}_{j}\right) \approx \mu\left(B_{j}\right)$ and

$$
\frac{2^{s j}}{C} \leq \frac{\mu\left(B_{j}\right)}{\mu\left(B_{0}\right)} \leq C 2^{t j}
$$

Using this, we obtain

$$
\mu_{a}\left(B_{a}\left(\infty, d_{a}(\infty, x)\right)\right) \approx \sum_{j=0}^{\infty} \frac{1}{\mu\left(B_{j}\right)}
$$

with

$$
\frac{1}{C \mu\left(B_{0}\right)} \sum_{j=0}^{\infty} 2^{-s j} \leq \sum_{j=0}^{\infty} \frac{1}{\mu\left(B_{j}\right)} \leq \frac{C}{\mu\left(B_{0}\right)} \sum_{j=0}^{\infty} 2^{-t j} .
$$

It follows from the assumption $d(x, a)>1 / C$ that

$$
\mu_{a}\left(B_{a}\left(\infty, d_{a}(x, \infty)\right)\right) \approx \frac{1}{\mu\left(B_{0}\right)}=\frac{1}{\mu(B(a, d(x, a)))} \approx \frac{1}{\mu(B(a, 1+d(x, a)))},
$$

that is, $d \mu_{a}^{\infty}(x) \approx d \mu(x)$ when $d(x, a)>1 / C$.

Case 2: $d(x, a) \leq 1 / C$. In this case, we have $1+d(x, a) \approx 1$, and so by the doubling property of $\mu$,

$$
\mu(B(a, 1+d(x, a))) \approx \mu(B(a, 1)) .
$$

For non-negative integers $j$, we now choose $B_{j}=B\left(a, 2^{j}\right)$. Then

$$
\begin{aligned}
\mu_{a}\left(B_{a}\left(\infty, d_{a}(x, \infty)\right)\right)= & \int_{X \backslash \bar{B}(a, d(x, a))} \frac{1}{\mu(B(a, 1+d(y, a)))^{2}} d \mu(y) \\
\approx & \int_{B(a, 1) \backslash \bar{B}(a, d(x, a))} \frac{1}{\mu(B(a, 1))^{2}} d \mu(y) \\
& +\sum_{j=0}^{\infty} \int_{B_{j+1} \backslash \bar{B}_{j}} \frac{1}{\mu(B(a, 1+d(y, a)))^{2}} d \mu(y) .
\end{aligned}
$$

Since $d(x, a) \leq 1 / C$, we have

$$
\mu(B(a, 1) \backslash \bar{B}(a, d(x, a))) \approx \mu(B(a, 1)),
$$

and for $y \in B_{j+1} \backslash B_{j}$ we also have that

$$
\mu(B(a, 1+d(y, a))) \approx \mu\left(B_{j}\right) .
$$

Hence

$$
\mu_{a}\left(B_{a}\left(\infty, d_{a}(x, \infty)\right)\right) \approx \frac{1}{\mu(B(a, 1))}+\sum_{j=0}^{\infty} \frac{\mu\left(B_{j+1} \backslash \bar{B}_{j}\right)}{\mu\left(B_{j}\right)^{2}}
$$


An application of (4) to the above now yields

$$
\mu_{a}\left(B_{a}\left(\infty, d_{a}(x, \infty)\right)\right) \approx \frac{1}{\mu(B(a, 1))} \approx \mu(B(a, 1+d(x, a))) .
$$

It now follows that $d \mu_{a}^{\infty}(x) \approx d \mu(x)$ even when $d(x, a) \leq 1 / C$.

This completes the proof of the lemma.

2.5. Radially star-like quasiconvex spaces and meridian-like quasiconvex spaces. The notions of radially star-like and meridian-like quasiconvexity were introduced in [9] to investigate the preservation of $\infty$-Poincaré inequality under the transformations of sphericalization and flattening.

Definition 2.8. A space is $K$-radially star-like quasiconvex with respect to a base point $a \in X$, if there exist a constant $K \geq 1$ and a radius $r_{0}>0$ such that for every $r>r_{0}$ and $x \in A(a, r / 2, r)$, there exist a base-point quasiconvex ray $\gamma_{a \infty}$, a point $y \in \gamma_{a \infty}$ and a quasiconvex curve $\gamma_{x y} \subset A(a, r / K, K r)$ connecting $x$ to $y$ such that

$$
\ell\left(\gamma_{x y}\right) \leq K d(a, y)
$$

Here we say that a ray $\gamma:[0, \infty) \rightarrow X$ with $\gamma(0)=a$ is base-point quasiconvex if for each $z \in|\gamma|, \ell\left(\gamma_{a z}\right) \leq C d(a, z)$, where $\gamma_{a z}$ is the subcurve of $\gamma$ ending at $z$.

Notice that if $(X, d)$ is a connected complete locally compact metric space which is annularly quasiconvex with respect to a point $a \in X$, then $(X, d)$ is $K$-radially star-like quasiconvex. See [9, Lemma 3.3].

Definition 2.9. A (bounded) metric space is $K$-meridian-like quasiconvex with respect to a base point $c \in X$, if there exists a constant $K \geq 1$, a point $a \in X$ and a small radius $r_{0}>0$ such that for every $x \in A(c, r / 2, r)$ with $r<r_{0}$, there exist a double base-point quasiconvex curve $\gamma_{a c}$, a point $y \in \gamma_{a c}$ and a curve $\gamma_{x y} \subset A(c, r / K, K r)$ connecting $x$ to $y$ such that

$$
\ell\left(\gamma_{x y}\right) \leq K d(y, c)
$$

By double base-point quasiconvex curve we mean that for any $z \in\left|\gamma_{a c}\right|$, $\ell\left(\gamma_{a z}\right) \leq C d(a, z)$ and $\ell\left(\gamma_{c z}\right) \leq C d(c, z)$. Here $\gamma_{a z}$ and $\gamma_{c z}$ denote the subcurves of $\gamma_{a c}$ with end points $a$ and $z$ and $c$ and $z$, respectively.

REMARK 1. The idea is to choose the point $a \in X$ (in Definition 2.9) in $A(c, R / 2, R)$ where $R=\sup _{z \in X} d(c, z)$. Then, $2 d(a, c) \geq \sup _{z} d(c, z) \geq$ $\operatorname{diam}(X) / 2$. Additionally, when $0<r \ll R$ and $x \in B(c, r)$, we have $d(x, a) \approx$ $d(a, c)$. Indeed, for $x \in B(c, r)$, we have that

$$
2 d(a, c)>d(a, c)+d(x, c) \geq d(a, x) \geq d(a, c)-d(x, c) \geq d(a, c)-r \approx d(a, c) .
$$


Observe that if $(X, d)$ is a bounded connected complete locally compact metric space which is annularly quasiconvex with respect to a point $c \in X$, then $(X, d)$ is $K$-meridian-like quasiconvex with respect to $c$. See $[9$, Lemma 4.3].

REMARK 2. It is possible to show that the sphericalization of an unbounded radially star-like quasiconvex space will result in a bounded meridian-like quasiconvex space, and vice versa. In fact, these two concepts are dual to each other via the dual transformations of sphericalization and flattening. See [9, Lemma 4.6, Lemma 4.7].

Unless otherwise stated, the letter $C$ denotes various positive constants whose exact values are not important for the purposes of this paper, and its value might change even within a line.

\section{Preservation of $p$-Poincaré inequality for $p>s$ under sphericalization}

Li and Shanmugalingam proved in [20, Theorem 3.3.5] the preservation of $p$ Poincaré inequality $(1 \leq p<\infty)$ under sphericalization for annularly quasiconvex spaces. In what follows, we show the preservation of $p$-Poincaré inequality under sphericalization for $p$ sufficiently large for metric spaces satisfying the radially star-like quasiconvexity property (see Definition 2.8). Metric spaces that are not annularly quasiconvex but are radially star-like quasiconvex are for example the real line, the Euclidean infinite strip $\mathbb{R} \times[-1,1]$, some classes of metric trees and Example 5.1 of the current paper.

THEOREM 3.1. Let $(X, d, \mu)$ be a complete unbounded metric space with a doubling measure $\mu$ so that $(X, d, \mu)$ supports a p-Poincaré inequality for some $p>s$, where $s$ is the exponent of relative lower volume decay associated to $\mu$ as in (2). Let $a \in X$ be a base point in $X$, and assume $(X, d)$ is $K$-radially star-like quasicovex with respect to a for some $K \geq 1$. Then $\left(\dot{X}, d_{a}, \mu_{a}\right)$ also supports a p-Poincaré inequality.

REMARK 3. Notice that we need $p>s$, which is associated to the exponent $s$ related to the original measure $\mu$ rather than the spherical measure $\mu_{a}$ from (2). See Example 1 below.

REMARK 4. The following heuristic argument could help to understand the main difficulty that arises when working with the weaker hypothesis of radially star-like quasiconvexity as opposed to working with annular quasiconvexity. Let $r<r_{0}$ and consider the ball $B_{a}(\infty, r)$. If the space is annularly quasiconvex, one can connect points in the same annulus with a curve that stays approximately in the same annulus. Under this hypothesis, it is therefore easy to compare points lying in the same horizontal annulus, that is, an annulus centered at $\infty$. On the other hand, radially star-like quasiconvexity 
allows us to connect any point $x$ to $\infty$ through a curve passing close to $x$, and $x$ connects to this curve through a quasiconvex curve. Therefore, in this case, it is easy to compare points that lie in the same vertical strip (denoted in the proof by $S_{i}$ ). Moreover, using the doubling property of the space, we will see that we can divide the ball into a finite number of vertical strips with the number independent of the radius of the ball. According to these two approaches, one could consider two possible divisions of the ball $B_{a}(\infty, r)$ :

$$
B_{a}(\infty, r)= \begin{cases}\bigcup_{i=0}^{\infty} A_{a}\left(\infty, 2^{-i-1} r, 2^{-i} r\right) & \text { division into "horizontal annuli", } \\ \bigcup_{i=0}^{N_{0}}\left(B_{a}(\infty, r) \cap S_{i}\right) & \text { division into "vertical strips". }\end{cases}
$$

In the proof that follows, we will use the division of the ball into "vertical strips".

Proof of Theorem 3.1. We need to verify $p$-Poincaré inequality for balls $B_{a}(x, r)$ with $x \in \dot{X}$ and $r>0$. We divide the proof into three different cases: balls far away from $\infty$ (whose behavior is similar to the balls in the original metric), balls centered at $\infty$, and more general balls. We assume $0<r<1 /\left(10 \lambda K^{2}\right)$, where $\lambda$ is the scaling constant involved in the $p$-Poincaré inequality and $K$ is the constant in the radially star-like quasiconvex property, because balls with radius $r \geq 1 /\left(10 \lambda K^{2}\right)$ can be compared to balls centered at $\infty$ with radius 1 , that is, balls that are equal to $\dot{X}$. Indeed, we will prove the $p$-Poincaré inequality for balls centered at $\infty$ in Case 2 without restricting the radius $r$ in that case.

Let $u \in \operatorname{Lip}(\dot{X})$ and let $g$ be an upper gradient of $u$ in $X$ with respect to the original metric $d$. Recall here that by Lemma 2.1 it suffices to verify the $p$-Poincaré inequality for functions $u$ that are Lipschitz continuous in $\dot{X}$.

Case 1: $d_{a}(x, \infty) \geq 8 \lambda r$. We can follow the same proof as the one of Case 1 in [20, Theorem 3.6]. Notice that the only hypotheses used in that proof are the doubling property of the measure $\mu$ (and $\mu_{a}$ ), and the fact that $X$ supports a $p$-Poincaré inequality (not the annular quasiconvexity property). We only recall here one estimate proven in Case 1 of [20, Theorem 3.6] that will be needed in Case 2. There is a positive integer $k_{0} \geq 3$ so that

$$
2^{k_{0}} \lambda r \leq d_{a}(x, \infty)=\frac{1}{1+d(x, a)} \leq 2^{k_{0}+1} \lambda r
$$

and there are two balls $B_{s}, B_{l}$ (with respect to the original metric) centered at $x$, with $B_{s} \subset B_{a}(x, r) \subset B_{l}$ and

$$
\frac{\mu\left(B_{s}\right)}{\mu\left(B\left(a, 1 /\left(2^{k_{0}} r\right)\right)\right)^{2}} \approx \mu_{a}\left(B_{a}(x, r)\right) \approx \frac{\mu\left(B_{l}\right)}{\mu\left(B\left(a, 1 /\left(2^{k_{0}} r\right)\right)\right)^{2}} .
$$

Using these balls the $p$-Poincaré inequality is proven for $B_{a}(x, r)$ on the left-hand side of the inequality and $B_{a}\left(x, \lambda_{0} r\right)$ on the right-hand side, with $\lambda_{0}$ depending solely on $\lambda$ and the doubling constant of $\mu$. 
Case 2: $x=\infty$ and $0<r<1 /\left(10 \lambda K^{2}\right)$. As shown in [9], because $X$ is radially star-like quasiconvex, $\dot{X}$ equipped with $d_{a}$ is a meridian-like quasiconvex space. Therefore, there exist a constant $K \geq 1$ and a fixed radius $r_{0}>0$ such that for every $R<r_{0}$ and $x \in A_{a}(\infty, R / 2, R)$, there exist a double base-point quasiconvex curve $\beta_{x}$ connecting $a$ to $\infty$ and a quasiconvex curve $\gamma_{x z} \subset A(\infty, R / K, K R)$ connecting $x$ to $z$ with $\ell_{d_{a}}\left(\gamma_{x z}\right) \leq K d_{a}(x, \infty)$.

In addition, in [20], it was shown that $\dot{X}$ is doubling. Therefore, if $\rho=\frac{r}{20 \lambda K^{2}}$, there exist $N_{0}=N_{0}\left(C_{\mu}\right)$ and sphericalized balls $\left\{B_{i}\right\}_{i=1}^{N_{0}}:=$ $\left\{B_{a}\left(z_{i}, \rho\right)\right\}_{i=1}^{N_{0}}$, such that $A_{a}(\infty, r / 2, r) \subset \bigcup_{i=1}^{N_{0}} B_{i}$ and $B_{i} \subset A_{a}(\infty, r / 3,2 r)$. The key point here is that the number of balls $N_{0}$ that cover the annulus depends only on the doubling constant and the ratio $\rho / r$, which is indeed independent of $r$.

Indeed, because $\dot{X}$ is endowed with a doubling measure $\mu_{a}$ with constant $C_{\mu_{a}}$, then the metric space $\left(\dot{X}, d_{a}\right)$ is doubling in the sense that there is a constant $N_{0}$ depending only on $C_{\mu_{a}}$ such that for each $r>0$, each closed ball in $\dot{X}$ of radius $2 r$ can be covered by a family of at most $N_{0}$ closed balls of radius $r$. Without loss of generality, we may assume that $3 r / 4 \leq d_{a}\left(z_{i}, \infty\right) \leq r$. Then the ball $B_{a}(\infty, r)$ can be written as a finite union of measurable sets, namely $B_{a}(\infty, r)=\bigcup_{i=1}^{N_{0}}\left(S_{i} \cap B_{a}(\infty, r)\right)$, where

$$
\begin{aligned}
S_{i}:= & \bigcup_{R<r_{0}}\left\{\in A_{a}(\infty, R / 2, R): \exists \beta_{y}\right. \text { double base-point quasiconvex curve } \\
& \text { connecting } a \text { to } \infty, \exists z \in \beta_{y} \text { and a quasiconvex curve } \\
& \left.\gamma_{y z} \subset A_{a}(\infty, R / K, K R) \text { with } \ell_{d_{a}}\left(\gamma_{y z}\right)<K d_{a}(y, \infty) \text { and } \beta_{y} \cap B_{i} \neq \emptyset\right\} .
\end{aligned}
$$

Here we write $R$ to distinguish it from the radius $r$ fixed above.

Notice that for every $x \in S_{i}$ there is a small neighborhood $N_{x}$ of $x$ with $N_{x} \subset S_{i}$, so $S_{i}$ is open in $\dot{X}$. Also, the intersection of two sets $S_{i}$ and $S_{j}, i \neq j$ could possibly be nonempty.

It might also be possible that $S_{i}=\emptyset$ for some $1 \leq i \leq N_{0}$ but, without loss of generality, we may assume $S_{i} \neq \emptyset$ for all $i$. If $S_{i} \neq \emptyset$, there exists a double base-point quasiconvex curve $\beta_{i}$ connecting $a$ to $\infty$ such that $\beta_{i} \cap B_{i} \neq \emptyset$. Let $y \in \beta_{i} \cap A_{a}(\infty, r / 2, r)$. Because $\dot{X}$ is quasiconvex (see [9, Theorem 3.4]), there exists $C_{0}$ such that $B_{a}\left(y, r / C_{0}\right) \subset S_{i}$ and so, by the doubling property of $\mu_{a}$, $\mu_{a}\left(S_{i} \cap B_{a}(\infty, r)\right) \approx \mu_{a}\left(B_{i}\right) \approx \mu_{a}\left(B_{a}(\infty, r)\right)$.

In addition, $B_{i}=B_{a}\left(z_{i}, \rho\right) \subset \frac{1}{3 K \lambda} B_{a}(\infty, 6 K \lambda r)$ and $K B_{a}(\infty, 6 K \lambda r) \subset$ $70 K^{3} \lambda B_{i}$.

Following the same argument as in Case 2 in [20, Theorem 3.4], we see that

$$
\int_{S_{i} \cap B_{a}(\infty, r)}\left|u-u_{B_{i}}\right| d \mu_{a} \leq C r\left(f_{\lambda B_{i}} \hat{g}^{p} d \mu_{a}\right)^{1 / p},
$$


where $\hat{g}$ is the upper gradient of $u$ in $\dot{X}$ defined in Lemma 2.4. We do not repeat here the argument but the idea is to control for each $1 \leq i \leq N_{0}$, the measure $\mu_{a}$ of the following level sets

$$
E_{t, i}=\left\{y \in S_{i} \cap B_{a}(\infty, r): y \neq \infty \text { and }\left|u(y)-u_{B_{i}}\right| \geq t\right\} .
$$

The only difference with the proof of [20, Case 2, Theorem 3.4] is that we use meridian-like quasiconvexity instead of annular quasiconvexity to connect $y \in S_{i} \cap B_{a}(\infty, r)$ with points in $B_{i}$. This is doable because, $y \in S_{i} \cap B_{a}(\infty, r)$ and $B_{i}$ are in the same "vertical strip". Notice that an annularly quasiconvex space is a meridian-like quasiconvex space with one single vertical strip (see $[9$, Lemma 4.5]).

Now observe that for a fixed $i_{0} \in\left\{1,2, \ldots, N_{0}\right\}$,

$$
\begin{aligned}
& \int_{B_{a}(\infty, r)}\left|u-u_{B_{a}(\infty, r)}\right| d \mu_{a} \\
& \leq 2 \int_{B_{a}(\infty, r)}\left|u-u_{B_{i_{0}}}\right| d \mu_{a} \\
& \leq 2 \sum_{i=1}^{N_{0}} \int_{S_{i} \cap B_{a}(\infty, r)}\left|u-u_{B_{i_{0}}}\right| d \mu_{a} \\
& \leq 2 \sum_{i=1}^{N_{0}} \int_{S_{i} \cap B_{a}(\infty, r)}\left(\left|u-u_{B_{i}}\right|+\left|u_{B_{i}}-u_{B_{i_{0}}}\right|\right) d \mu_{a} \\
& \leq 2 \sum_{i=1}^{N_{0}} \int_{S_{i} \cap B_{a}(\infty, r)}\left|u-u_{B_{i}}\right| d \mu_{a} \\
& \quad+2 \sum_{i=1}^{N_{0}} \mu_{a}\left(S_{i} \cap B_{a}(\infty, r)\right)\left|u_{B_{i}}-u_{B_{i_{0}}}\right| .
\end{aligned}
$$

Notice that the first summand of the last inequality can be estimated by using (6) as follows:

$$
\begin{aligned}
& \sum_{i=1}^{N_{0}} \int_{S_{i} \cap B_{a}(\infty, r)}\left|u-u_{B_{i}}\right| d \mu_{a} \\
& \quad \leq C r \sum_{i=1}^{N_{0}} \mu_{a}\left(B_{i}\right)\left(f_{\lambda B_{i}} \hat{g}^{p} d \mu_{a}\right)^{1 / p} \\
& \quad \approx C N_{0} \mu_{a}\left(B_{a}(\infty, r)\right) r\left(f_{\lambda B_{a}(\infty, r)} \hat{g}^{p} d \mu_{a}\right)^{1 / p} .
\end{aligned}
$$

The second summand of inequality (7) can be estimated by using the point $x=\infty$. For that, we need to compare $B_{i}$ with $B_{i_{0}}$, balls that lie in the same "horizontal annulus". Since we only know how to compare points in a 
"vertical strip", one has to compare first $B_{i}$ with the point at $\infty$ (lying both in a "vertical strip") and then $\infty$ with $B_{i_{0}}$ (lying both in another "vertical strip") as the following inequality illustrates:

$$
\begin{aligned}
& \sum_{i=1}^{N_{0}} \mu_{a}\left(S_{i} \cap B_{a}(x, r)\right)\left|u_{B_{i}}-u_{B_{i_{0}}}\right| \\
& \quad \leq \sum_{i=1}^{N_{0}} \mu_{a}\left(S_{i} \cap B_{a}(\infty, 2 r)\right)\left(\left|u_{B_{i}}-u(\infty)\right|+\left|u(\infty)-u_{B_{i_{0}}}\right|\right) .
\end{aligned}
$$

Now, fix $1 \leq i \leq N_{0}$.

First find $z_{i, 0} \in \beta_{i}$, where $\beta_{i}$ is a ray in $S_{i}$, such that $d_{a}\left(\infty, z_{i, 0}\right)=d_{a}\left(\infty, z_{i}\right)$ and denote $B_{i, 0}=B_{i}$. We can choose a sequence of points $z_{i, j} \in \beta_{i}$ by induction to estimate $\left|u_{B_{i}}-u(\infty)\right|$. Suppose $z_{i, j-1}$ has been chosen, with $z_{i, j-1} \in A_{a}\left(\infty, 2^{-l_{j-1}-1} r, 2^{-l_{j-1}} r\right)$, where $l_{j-1}$ is an integer depending only on $j$. We can find a point $z_{i, j}$ in the subcurve of $\beta_{i}$ connecting $z_{i, j-1}$ to $\infty$, denoted by $\beta_{\infty z_{i, j-1}}$, such that the length of the subcuve $\gamma_{i, j}$ of $\beta_{\infty z_{i, j-1}}$ with end points $z_{i, j-1}$ and $z_{i, j}$ satisfies $2^{-l_{j-1}-1} \rho \leq \ell_{d_{a}}\left(\gamma_{i, j}\right) \leq 2^{-l_{j-1}} \rho$. Since $d_{a}\left(z_{i, j-1}, \infty\right) \geq 2^{-l_{j-1}-1} r \geq 2^{-l_{j-1}} \rho \geq \ell_{d_{a}}\left(\gamma_{i, j}\right)$, such $z_{i, j}$ always exists. Once $z_{i, j}$ has been chosen, we can choose $z_{i, j+1}$ in the subcurve of $\beta_{i}$ connecting $z_{i, j}$ to $\infty$ satisfying $2^{-l_{j}-1} \rho \leq \ell_{d_{a}}\left(\gamma_{i, j+1}\right) \leq 2^{-l_{j}} \rho$, where $\gamma_{i, j+1}$ can be defined as before. Therefore, we have chosen a sequence of points $z_{i, j} \in \beta_{i}$.

We now need to prove that

$$
\lim _{j \rightarrow \infty} d_{a}\left(z_{i, j}, \infty\right)=0
$$

Let $N_{l}:=\left\{j \in \mathbb{N}: l_{j}=l\right\}$. We first need to show that for every $l \geq 0$, we have $\#\left(N_{l}\right) \leq M(K, \lambda)$. Let $s_{l}=\min j \in N_{l}$. By the base-point quasiconvexity of $\beta_{i}$ with respect to base point $\infty$, we have

$$
\begin{aligned}
\#\left(N_{l}\right) 2^{-l-1} \rho & =\sum_{j \in N_{l}} 2^{-l_{j}-1} \rho \leq \sum_{j=s_{l}}^{\infty} 2^{-l_{j}-1} \rho \leq \sum_{j=s_{l}}^{\infty} \ell_{d_{a}}\left(\gamma_{i, j+1}\right) \\
& \leq \ell_{d_{a}}\left(\beta_{\infty z_{i, s_{l}}}\right) \leq C d_{a}\left(z_{i, s_{l}}, \infty\right) \\
& \leq 2^{-l_{s_{l}}} r=2^{-l} r
\end{aligned}
$$

so $\#\left(N_{l}\right) \leq M$ for some $M=M(K, \lambda)$. Hence, for each $l \geq 0$, there exists $j \in \mathbb{N}$ so that when $j \geq M l$, we have $l_{j} \geq l$, and so it follows that $\lim _{j \rightarrow \infty} d_{a}\left(z_{i, j}, \infty\right) \leq \lim _{j \rightarrow \infty} 2^{-l_{j}} r=0$. 
Now we can take the collection of sphericalized balls $B_{i, j}=B_{a}\left(z_{i, j}, 2^{-l_{j}} \rho\right)$ to estimate $\left|u_{B_{i}}-u(\infty)\right|$. Notice that $\operatorname{rad}\left(B_{i, j}\right)$ tends to zero when $j$ approaches to $\infty$. Then we can obtain the estimate as follows:

$$
\begin{aligned}
& \left|u_{B_{i}}-u(\infty)\right| \\
& \quad \leq \sum_{j=0}^{\infty}\left|u_{B_{i, j}}-u_{B_{i, j+1}}\right| \leq 4 \sum_{j=0}^{\infty} \int_{2 B_{i, j}}\left|u-u_{2 B_{i, j}}\right| d \mu_{a} \\
& \quad \leq C \sum_{j=0}^{\infty} \frac{\operatorname{rad}_{a}\left(2 B_{i, j}\right)}{\mu_{a}\left(2 B_{i, j}\right)^{1 / p}}\left(\int_{6 \lambda K^{2} B_{i, j}} \hat{g}^{p} d \mu_{a}\right)^{1 / p} \\
& \left.\quad \leq C\left(\sum_{j=0}^{\infty}\left(\frac{\operatorname{rad}_{a}\left(2 B_{i, j}\right)}{\mu_{a}\left(2 B_{i, j}\right)^{1 / p}}\right)^{\frac{p}{p-1}}\right)^{\frac{p-1}{p}}\left(\sum_{j=0}^{\infty} \int_{6 \lambda K^{2} B_{i, j}} \hat{g}^{p} d \mu_{a}\right)^{1 / p} \hat{g}^{p} d \mu_{a}\right)^{1 / p}, \\
& \quad \leq C\left(\sum_{j=0}^{\infty}\left(\frac{\operatorname{rad}_{a}\left(B_{i, j}\right)}{\mu_{a}\left(B_{i, j}\right)^{1 / p}}\right)^{\frac{p}{p-1}}\right)^{\frac{p-1}{p}}\left(M \int_{6 \lambda K^{2}\left(S_{i} \cap B_{a}(x, r)\right)}\right.
\end{aligned}
$$

where in the third line we have used Hölder inequality and in the second line we have applied the $p$-Poincaré inequality for balls $B_{i, j}$, which satisfy the hypothesis of Case 1. Here $\operatorname{rad}_{a}(B)$ is the radius of $B$ in the metric $d_{a}$.

On the other hand, we need to estimate the quantity $\left(\operatorname{rad}_{a}\left(B_{i, j}\right)\right) /$ $\left(\mu_{a}\left(B_{i, j}\right)\right)$. Since $\operatorname{rad}_{a}\left(B_{i, j}\right)=2^{-l_{j}} \rho$ and $d_{a}\left(z_{i, j}, \infty\right) \geq 2^{-l_{j}-1} r$, by $(5)$, we have

$$
\mu_{a}\left(B_{i, j}\right) \approx \frac{\mu\left(B\left(z_{i, j}, C 2^{l_{j}} / \rho\right)\right)}{\mu\left(B\left(a, C 2^{l_{j}} / \rho\right)\right)^{2}}
$$

and

$$
\mu_{a}\left(B_{i}\right) \approx \frac{\mu\left(B\left(z_{i}, c / \rho\right)\right)}{\mu(B(a, c / \rho))^{2}} .
$$

Therefore by (2), we have

$$
\begin{aligned}
\frac{\mu_{a}\left(B_{i, j}\right)}{\mu_{a}\left(B_{i}\right)} & \approx \frac{\mu\left(B\left(z_{i, j}, c 2^{l_{j}} / \rho\right)\right) \mu(B(a, c / \rho))^{2}}{\mu\left(B\left(z_{i}, c / \rho\right)\right) \mu\left(B\left(a, c 2^{l_{j}} / \rho\right)\right)^{2}} \approx \frac{\mu(B(a, c / \rho))}{\mu\left(B\left(a, c 2^{l_{j}} / \rho\right)\right)} \\
& \geq C^{-1}\left(\frac{c / \rho}{c 2^{l_{j}} / \rho}\right)^{s} \\
& \approx C\left(\frac{2^{-l_{j}} \rho}{\rho}\right)^{s},
\end{aligned}
$$

and so it follows that

$$
\frac{\left(2^{-l_{j}} \rho\right)^{s / p}}{\mu_{a}\left(B_{i, j}\right)^{1 / p}} \leq C \frac{\rho^{s / p}}{\left(\mu_{a}\left(B_{a}\left(z_{i}, \rho\right)\right)\right)^{1 / p}} .
$$


Then we obtain the upper bound of the first term in the last inequality of (11), which is

$$
\begin{aligned}
& \left(\sum_{j=0}^{\infty}\left(\frac{\operatorname{rad}_{a}\left(B_{i, j}\right)}{\mu_{a}\left(B_{i, j}\right)^{1 / p}}\right)^{\frac{p}{p-1}}\right)^{\frac{p-1}{p}} \\
& \quad=\left(\sum_{j=0}^{\infty}\left(\frac{\left(2^{-l_{j}} \rho\right)^{s / p}\left(2^{-l_{j}} \rho\right)^{1-s / p}}{\mu_{a}\left(B_{i, j}\right)^{1 / p}}\right)^{\frac{p}{p-1}}\right)^{\frac{p-1}{p}} \\
& \leq\left(\sum_{j=0}^{\infty}\left(\frac{\rho^{s / p}\left(2^{-l_{j}} \rho\right)^{1-s / p}}{\mu_{a}\left(B_{i}\right)^{1 / p}}\right)^{\frac{p}{p-1}}\right)^{\frac{p-1}{p}} \\
& \quad=\frac{\rho}{\left(\mu_{a}\left(B_{i}\right)\right)^{1 / p}}\left(\sum_{j=0}^{\infty} 2^{-l_{j} \frac{p-s}{p-1}}\right)^{\frac{p-1}{p}}
\end{aligned}
$$

Notice from the argument of (10) and the subsequent paragraph that for each $k \in \mathbb{N}$, there are at most $M$ number of $j$ with $l_{j}=k$. So the quantity $\sum_{j=0}^{\infty} 2^{-l_{j} \frac{p-s}{p-1}}$ is finite. Combining (11) and (12), we obtain that

$$
\left|u_{B_{i}}-u(\infty)\right| \leq C r\left(f_{6 \lambda K^{2}\left(S_{i} \cap B_{a}(x, r)\right)} \hat{g}^{p} d \mu_{a}\right)^{1 / p} .
$$

Combining (7),(8), (9) and the inequality above, we obtain that

$$
f_{B_{a}(\infty, r)}\left|u-u_{B_{a}(\infty, r)}\right| d \mu_{a} \leq C r\left(f_{6 \lambda K^{2} B_{a}(\infty, r)} \hat{g}^{p} d \mu_{a}\right)^{1 / p}
$$

as wanted.

Case 3: $d_{a}(x, \infty)<8 \lambda r$. In this case, we use the conclusion of Case 2 above as an aid, since $B_{a}(x, r) \subset B_{a}(\infty, 16 \lambda r), B_{a}\left(\infty, 96 K \lambda^{2} r\right) \subset B_{a}\left(x, 105 K \lambda^{2} r\right)$ and the ball $B_{a}(\infty, 16 \lambda r)$ satisfies the hypothesis of Case 2. Hence by the doubling property of $\mu_{a}$,

$$
\begin{aligned}
f_{B_{a}(x, r)}\left|u-u_{B_{a}(x, r)}\right| d \mu_{a} & \leq 2 f_{B_{a}(x, r)}\left|u-u_{B_{a}(\infty, 16 \lambda r)}\right| d \mu_{a} \\
& \leq C f_{B_{a}(\infty, 16 \lambda r)}\left|u-u_{B_{a}(\infty, 16 \lambda r)}\right| d \mu_{a} \\
& \leq C r\left(f_{96 K \lambda^{2} B_{a}(\infty, r)} \hat{g}^{p} d \mu_{a}\right)^{1 / p} \\
& \leq C r\left(f_{B_{a}\left(x, 105 K \lambda^{2} r\right)} \hat{g}^{p} d \mu_{a}\right)^{1 / p} .
\end{aligned}
$$


The following example was considered in [20] and shows that the previous theorem is not true for $p \leq s$.

ExAmple 1. Let $X$ be the 2-dimensional Euclidean strip $\mathbb{R} \times[-1,1]$ equipped with the Euclidean metric and the weighted measure $d \mu(x)=$ $\max \left\{1,|x|^{2}\right\} d \mathscr{L}^{2}(x)$, which is clearly a radially star-like quasiconvex space. By [14, Corollary 15.35] the measure $\mu$ is $p$-admissible in $\mathbb{R}^{2}$ for any $p>1$, which means that $\mu$ is doubling and $\left(\mathbb{R}^{2},|\cdot|, \mu\right)$ supports a $p$-Poincaré inequality for any $p>1$. In particular $(X,|\cdot|, \mu)$ supports a 2-Poincaré inequality. The sphericalized space with respect to the base point $a=(0,0)$ is the region trapped between two tangential circles in the sphere. In particular, the boundary of such a region is a quadratic cusp and one can check that $\mu_{a}=\mathscr{L}^{2}$. Therefore, $\left(X_{a}, d_{a}, \mu_{a}\right)$ supports a $p$-Poincaré inequality for any $p>3$. Observe that in this case the exponent of relative lower volume decay associated to $\mu$ is $s=3$.

On the other hand, [9, Example 3.14] provides a metric measure space endowed with a doubling measure, which is not radially star-like quasiconvex, supporting an $\infty$-Poincaré inequality but whose sphericalized space fails to support an $\infty$-Poincaré inequality. We can therefore conclude that Theorem 3.1 is no longer true if the hypothesis of radially star-like quasiconvexity is removed.

\section{Preservation of $p$-Poincaré inequality for $p>s$ under flattening}

In this section, we show the preservation of $p$-Poincaré inequality under flattening for $p$ sufficiently large for meridian-like quasiconvex metric spaces supporting a doubling measure (see Definition 2.9).

TheOREM 4.1. Let $(X, d, \mu)$ be a bounded complete metric space endowed with a doubling measure $\mu$ and supporting a p-Poincaré inequality for some $p>s$, where $s$ is the exponent of relative lower volume decay associated to $\mu$ as in (2). Let $c \in X$ be a base point on $X$, and assume $(X, d)$ is $K$-meridian-like quasiconvex with respect to the base point $c$ for some $K \geq 1$. Then $\left(X^{c}, d^{c}, \mu^{c}\right)$ also supports a p-Poincaré inequality.

REMARK 5. Notice that we require $p>s$, where $s$ is associated to the original measure $\mu$ rather than the flattened measure $\mu^{c}$.

Proof of Theorem 4.1. Let $u \in \operatorname{Lip}\left(X^{c}\right)$ and $g$ be an upper gradient of $u$ in $X$ with respect to the metric $(X, d)$. We split the proof into three different cases depending on the quantity $\lambda r d(x, c)$.

In the first case, we consider balls $B^{c}(x, r)$ far away from the singular point $c$ (with a behaviour similar to the original ball). In the second case, the flattened ball $B^{c}(x, r)$ behaves like the complement of an unflattened ball centered at $c$. In the third case, we cover the rest of possibilities. 
As shown in [9], because $X$ is meridian-like quasiconvex, $X^{c}$ equipped with $d^{c}$ is radially star-like quasiconvex. Therefore, there exist a constant $K \geq 1$, a fixed radius $r_{0}>0$ and a point $a \in X$ (as explained in Remark 1) such that for every $R>r_{0}$ and $y \in A^{c}(a, R / 2, R)$, there exist a base-point quasiconvex ray $\beta_{y}$ connecting $a$ to $\infty$, a point $z \in \beta_{y}$ and a quasiconvex curve $\gamma_{y z} \subset A^{c}(a, R / K, K R)$ connecting $y$ to $z$ with $\ell_{d^{c}}\left(\gamma_{y z}\right) \leq K d^{c}(a, z)$. Denote $L=\max \left\{r_{0} d(a, c) / 4,1\right\}$, a constant that will be used in Case 2 and Case 3 .

Case 1: $6 \lambda r d(x, c) \leq 1 / 2$. This case is the same as Case 1 of [20, Theorem 4.4]. Observe that in that proof one only uses the $p$-Poincaré inequality (not the annularly quasiconvexity property) and the doubling property of the measure $\mu$, so the same argument works in our setting.

Case 2: $\lambda r d(x, c) \geq 4 \lambda L$. According to Case 2 of [20, Theorem 4.4], we can see that

$$
X \backslash \bar{B}(c, 2 / r) \subset B^{c}(x, r) \subset X \backslash \bar{B}(c, 2 /(3 r)) .
$$

First, we assume that $x=a$.

In [20], it was shown that $X^{c}$ is doubling. Therefore, if $\rho=r /(96 \lambda K)$ there exist $N_{0}=N_{0}\left(C_{\mu}\right)$ flattened balls $\left\{B_{i}\right\}_{i=1}^{N_{0}}:=\left\{B^{c}\left(z_{i}, \rho\right)\right\}_{i=1}^{N_{0}}$, such that $A^{c}(a, r / 2, r) \subset \bigcup_{i=1}^{N_{0}} B_{i}$, with $r d\left(z_{i}, c\right) \approx 4 L$. Then the ball $B^{c}(a, r)$ can be written as a finite union of measurable sets, namely $B^{c}(a, r)=$ $\bigcup_{i=1}^{N_{0}}\left(S_{i} \cap B^{c}(a, r)\right)$, where

$$
\begin{aligned}
S_{i}:= & \bigcup_{R>r_{0}}\left\{y \in A^{c}(a, R / 2, R): \exists \beta_{y}\right. \text { base-point quasiconvex ray } \\
& \text { connecting } a \text { to } \infty, \exists z \in \beta_{y} \text { and a quasiconvex curve } \\
& \left.\gamma_{y z} \subset A^{c}(a, R / K, K R) \text { with } \ell_{d^{c}}\left(\gamma_{y z}\right)<K d^{c}(a, y) \text { and } \beta_{y} \cap B_{i} \neq \emptyset\right\} .
\end{aligned}
$$

Notice that for every $x \in S_{i}$ there is a small neighborhood $N_{x}$ of $x$ with $N_{x} \subset S_{i}$, so $S_{i}$ is open in $X^{c}$.

As we did in Case 2 of Theorem 3.1, we assume $S_{i} \neq \emptyset$. If $S_{i} \neq \emptyset$, there exists a base-point quasiconvex ray $\beta_{i}$ connecting $a$ to $\infty$ such that $\beta_{i} \cap B_{i} \neq \emptyset$. Now, let $y \in \beta_{i} \cap A^{c}(a, r / 2, r)$. Because $X^{c}$ is quasiconvex (see [9, Theorem 4.10.]), there exists $C_{0}$ such that $B^{c}\left(y, r / C_{0}\right) \subset S_{i}$ and so, by the doubling property of $\mu^{c}$, we have that $\mu^{c}\left(S_{i} \cap B^{c}(a, r)\right) \approx \mu^{c}\left(B_{i}\right) \approx \mu^{c}\left(B^{c}(a, r)\right)$.

Observe that the radius of the ball $B_{i}=B^{c}\left(z_{i}, \rho\right)$ satisfies the hypothesis of Case 1 , that is, $6 \lambda \rho d\left(z_{i}, c\right)=6 \lambda d\left(z_{i}, c\right) r /(96 \lambda K) \leq 1 / 2$.

Following the same argument as in Case 2 of [20, Theorem 4.4.], we see that

$$
f_{S_{i} \cap B^{c}(a, r)}\left|u-u_{B_{i}}\right| d \mu^{c} \leq C r\left(f_{6 \lambda K B^{c}(a, r)} \bar{g}^{p} d \mu^{c}\right)^{1 / p}
$$

where $\bar{g}$ is the upper gradient of $u$ in $X^{c}$ defined in Lemma 2.4. Again, the only difference with the proof of [20, Case 2, Theorem 4.3.3] is that we use radially star-like quasiconvexity instead of annular quasiconvexity to connect 
$y \in S_{i} \cap B^{c}(a, r)$ with points in $B_{i}$. We can do this because $y \in S_{i} \cap B^{c}(a, r)$ and $B_{i}$ are in the same "vertical strip". Next, observe that

$$
\begin{aligned}
& \int_{B^{c}(x, r)}\left|u-u_{B^{c}(a, r)}\right| d \mu^{c} \\
& \quad \leq 2 \int_{B^{c}(a, r)}\left|u-u_{B_{1}}\right| d \mu^{c} \leq 2 \sum_{i=1}^{N_{0}} \int_{S_{i} \cap B^{c}(a, r)}\left|u-u_{B_{1}}\right| d \mu^{c} \\
& \quad \leq \sum_{i=1}^{N_{0}} \int_{S_{i} \cap B^{c}(a, r)}\left(\left|u-u_{B_{i}}\right|+\left|u_{B_{i}}-u_{B_{1}}\right|\right) d \mu^{c} \\
& \quad \leq \sum_{i=1}^{N_{0}} \int_{S_{i} \cap B^{c}(a, r)}\left|u-u_{B_{i}}\right|+\sum_{i=1}^{N_{0}} \mu^{c}\left(S_{i} \cap B^{c}(a, r)\right)\left|u_{B_{i}}-u_{B_{1}}\right| .
\end{aligned}
$$

Notice that we can estimate the first summand of the last inequality by using (13), so we only need to estimate the second summand.

Since $d(a, c) \approx \sup _{z \in X} d(z, c)$, there exists $l \geq 0$ with $2^{l} d\left(z_{i}, c\right) \leq d(a, c)<$ $2^{l+1} d\left(z_{i}, c\right)$. In what follows, denote $z_{i, 0}=z_{i}, z_{i, M_{i}}=a$ where $M_{i}$ will be shown to be bounded in the next paragraph. Then similar to Case 2 of Theorem 3.1, we can construct a collection of points $z_{i, k}$, where $k=0,1,2, \ldots, M_{i}$ from $\beta_{i}$ by induction. Suppose $z_{i, k-1}$ has been chosen. Then denote $\beta_{z_{i, k-1} a}$ to be the subcurve of $\beta_{i}$ connecting $z_{i, k-1}$ to $a$, and $z_{i, k-1} \in$ $A\left(c, 2^{l_{k}-1} d\left(z_{i}, c\right), 2^{l_{k}} d\left(z_{i}, c\right)\right)$ (with respect to the metric $d$ ), where $l_{k}$ is an integer depending only on $k$. We can find a point $z_{i, k} \in \beta_{z_{i, k-1} a}$ such that the length of the subcurve $\gamma_{i, k}$ of $\beta_{i}$ connecting $z_{i, k-1}$ to $z_{i, k}$ satisfies $2^{-l_{k-1}-1} \rho \leq \ell^{c}\left(\gamma_{i, k}\right) \leq 2^{-l_{k-1}} \rho$.

Let $N_{s}=\left\{j \leq M_{i}: l_{j}=s\right\}$. We first need to show that for each $s \leq l$, $\#\left(N_{s}\right) \leq M$ for $M=M(K, \lambda)$. Let $j_{s}=\min j \in N_{s}$. Because $(X, d)$ is meridian-like quasiconvex, $\beta_{i}$ is a base-point quasicovex ray with respect to the point $a$ and the metric $d^{c}$ and so we have

$$
\begin{aligned}
\#\left(N_{s}\right) 2^{-s-1} \rho & =\sum_{j \in N_{s}} 2^{-l_{j}} \rho \leq \sum_{j \in N_{s}} \ell^{c}\left(\gamma_{i, j}\right) \leq \sum_{j=j_{s}}^{M_{l}} \ell^{c}\left(\gamma_{i, j}\right) \\
& =\ell^{c}\left(\beta_{z_{i, j_{s}} a}\right) \leq C d^{c}\left(a, z_{i, j_{s}}\right) .
\end{aligned}
$$

Since $d^{c}\left(a, z_{i, j_{s}}\right)=\frac{d\left(a, z_{i, j_{s}}\right)}{d(a, c) d\left(z_{i, j_{s}}, c\right)}$ and $d\left(a, z_{i, j_{s}}\right) \leq d(a, c)+d\left(z_{i, j_{s}}, c\right) \leq$ $2 d(a, c)$, so

$$
d^{c}\left(a, z_{i, j_{s}}\right) \leq \frac{2}{d\left(z_{i, j_{s}}, c\right)} \leq \frac{2}{2^{l_{j_{s}}-1} d\left(z_{i}, c\right)}=2^{-s} 16 r, \quad \text { for } r d\left(z_{i}, c\right)=4 .
$$

Therefore, we have $\#\left(N_{s}\right) \leq M$ and also $M_{i} \leq \sum_{s=0}^{l} \#\left(N_{s}\right) \leq M(l+1)$. 
Set $\rho_{k}=2^{-j_{k}} \rho$. Then we can construct a collection of flattened balls $B_{i, k}=$ $B^{c}\left(z_{i, k}, \rho_{k}\right)$ in order to estimate the second summand in (14). Note that

$$
\left|u_{B_{i}}-u_{B_{1}}\right| \leq\left|u_{B_{i}}-u_{B_{i, M_{i}}}\right|+\left|u_{B_{i, M_{i}}}-u_{B_{1}}\right|
$$

Without loss of generality, it suffices to estimate $\left|u_{B_{i, M_{i}}}-u_{B_{i}}\right|$. To estimate $\left|u_{B_{i, M_{i}}}-u_{B_{i}}\right|$, notice that $u_{B_{i, M_{i}}}=u_{B_{1, M_{1}}}$ for $i=1,2, \ldots, N_{0}$, so $\left|u_{B_{i, M_{i}}}-u_{B_{1}}\right|=\left|u_{B_{1, M_{1}}}-u_{B_{1}}\right|$.

Then we have

$$
\begin{aligned}
& \left|u_{B_{i}}-u_{B_{i, M_{i}}}\right| \\
& \leq \sum_{k=1}^{M_{i}}\left|u_{B_{i, k}}-u_{B_{i, k+1}}\right| \leq 2 \sum_{k=1}^{M_{i}} \int_{2 B_{i, k}}\left|u-u_{B_{i, k}}\right| d \mu^{c} \\
& \quad \leq C \sum_{k=1}^{M_{i}} \frac{\operatorname{rad}\left(2 B_{i, k}\right)}{\mu^{c}\left(2 B_{i, k}\right)^{1 / p}}\left(\int_{6 \lambda K B_{i, k}} \bar{g}^{p} d \mu^{c}\right)^{1 / p} \\
& \leq C\left(\sum_{k=1}^{M_{i}}\left(\frac{\operatorname{rad}\left(2 B_{i, k}\right)}{\mu^{c}\left(2 B_{i, k}\right)^{1 / p}}\right)^{p /(p-1)}\right)^{(p-1) / p}\left(\int_{6 \lambda K B_{j, k}} \bar{g}^{p} d \mu^{c}\right)^{1 / p} \\
& \leq C\left(\sum_{k=1}^{M_{i}}\left(\frac{\operatorname{rad}\left(2 B_{i, k}\right)}{\mu^{c}\left(2 B_{i, k}\right)^{1 / p}}\right)^{p /(p-1)}\right)^{(p-1) / p}\left(C_{6 \lambda K S_{i}} \bar{g}^{p} d \mu^{c}\right)^{1 / p},
\end{aligned}
$$

where in the third line we have used Hölder inequality and the fact that, by the doubling assumption on $\mu, \mu^{c}$ is also doubling. In the second line, we have applied the $p$-Poincaré inequality for the balls $B_{i, k}$ which satisfy the hypothesis of Case 1 . Indeed, recall that $B_{i, k}=B^{c}\left(z_{i, k}, \rho_{k}\right)$, and $2^{j_{k}} d\left(z_{i}, c\right) \leq d\left(z_{j, k}, c\right) \leq 2^{j_{k}+1} d\left(z_{i}, c\right), \rho_{k}=2^{-j_{k}} \rho=2^{-j_{k}} r /(96 \lambda K)$, so $d\left(z_{j, k}, c\right) \rho_{j} \leq 2 d\left(z_{i}, c\right) r /(96 \lambda K)=1 /(12 \lambda K)$.

Now, according to Case 1 , since $6 \lambda \operatorname{rad}\left(B_{i, k}\right) d\left(z_{i, k}, c\right) \leq 1 / 2$, then we have

$$
\begin{aligned}
\mu^{c}\left(B^{c}\left(z_{i, k}, \rho_{k}\right)\right) & =\int_{B^{c}\left(z_{i, k}, \rho_{k}\right)} \frac{d \mu(y)}{\mu(B(c, d(y, c)))^{2}} \approx \frac{\mu\left(B^{c}\left(z_{i, k}, \rho_{k}\right)\right)}{\mu\left(B\left(c, d\left(z_{i, k}, c\right)\right)\right)^{2}} \\
& \approx \frac{\mu\left(B\left(z_{i, k}, \rho_{k} d\left(z_{i, k}, c\right)^{2}\right)\right)}{\mu\left(B\left(c, d\left(z_{i, k}, c\right)\right)\right)^{2}}
\end{aligned}
$$

Notice that $\rho_{k} d\left(z_{i, k}, c\right) \approx \rho d\left(z_{i}, c\right)=1 /(12 \lambda K) \leq 1 / 2$. By the doubling property, of $\mu$ we have

$$
C_{\mu} \geq \frac{\mu\left(B\left(z_{i, k}, \rho_{k} d\left(z_{i, k}, c\right)^{2}\right)\right)}{\mu\left(B\left(c, d\left(z_{i, k}, c\right)\right)\right)} \geq C \frac{\left(\frac{d\left(z_{i, k}, c\right)}{24 \lambda K}\right)^{s}}{d\left(z_{i, k}, c\right)^{s}} \geq \frac{1}{C}
$$


Therefore, from the estimate above and (17) we can induce that

$$
\begin{aligned}
\frac{\mu^{c}\left(B^{c}\left(z_{i, k}, \rho_{k}\right)\right)}{\mu^{c}\left(B^{c}\left(z_{i}, \rho\right)\right)} & \approx \frac{\mu\left(B\left(z_{i, k}, \rho_{k} d\left(z_{i, k}, c\right)^{2}\right)\right)}{\mu\left(B\left(c, d\left(z_{i, k}, c\right)\right)\right)^{2}} \frac{\mu\left(B\left(c, d\left(z_{i}, c\right)\right)\right)^{2}}{\mu\left(B\left(z_{i}, \rho d\left(z_{i}, c\right)^{2}\right)\right)} \\
& \approx \frac{\mu\left(B\left(c, d\left(z_{i}, c\right)\right)\right)}{\mu\left(B\left(c, d\left(z_{i, k}, c\right)\right)\right)} \geq C\left(\frac{d\left(z_{i}, c\right)}{d\left(z_{i, k}, c\right)}\right)^{s} \approx C\left(\frac{(4 / r)}{2^{j_{k}}(4 / r)}\right)^{s} .
\end{aligned}
$$

Hence, we can get

$$
\frac{\left(2^{-j_{k}} r\right)^{s / p}}{\mu^{c}\left(B_{i, k}\right)^{1 / p}} \leq C \frac{r^{s / p}}{\mu^{c}\left(B^{c}\left(z_{i}, \rho\right)\right)^{1 / p}} .
$$

From this estimate together with (16), we obtain

$$
\begin{aligned}
& \left(\sum_{k=1}^{M_{i}}\left(\frac{\operatorname{rad}\left(2 B_{i, k}\right)}{\mu^{c}\left(2 B_{i, k}\right)^{1 / p}}\right)^{\frac{p}{(p-1)}}\right)^{\frac{(p-1)}{p}} \\
& \quad \leq C\left(\sum_{k=1}^{M_{i}}\left(\frac{\left(2^{-j_{k}} r\right)^{s / p}\left(2^{-j_{k}} r\right)^{1-s / p}}{\left(\mu^{c}\left(2 B_{i, k}\right)^{1 / p}\right)}\right)^{\frac{p}{(p-1)}}\right)^{\frac{(p-1)}{p}} \\
& \left.\leq C\left(\sum_{k=1}^{M_{i}}\left(\frac{r^{s / p}\left(2^{-j_{k}} r\right)^{1-s / p}}{\left(\mu^{c}\left(B_{i}\right)^{1 / p}\right)}\right)^{\frac{p}{(p-1)}}\right)^{\frac{(p-1)}{p}}\right)^{\frac{(p-1)}{p}} \frac{r}{\mu^{c}\left(B_{i}\right)^{1 / p}} .
\end{aligned}
$$

From (18), we can go back to (16), then we can derive that

$$
\left|u_{B_{i}}-u_{B_{i, M_{i}}}\right| \leq C \frac{r}{\mu^{c}\left(B_{i}\right)^{1 / p}}\left(\int_{6 \lambda K S_{i}} \bar{g}^{p} d \mu^{c}\right)^{1 / p} .
$$

Combining with (14), (15) and (19), we have proved Case 2 for $x=a$.

If $x \neq a$, because $2 d(a, c) \geq \sup _{z \in X} d(z, c)$ and $r d(x, c) \geq 4 L$ we have that $r d(a, c) \geq 2 L$. We can observe that the balls $B^{c}(a, r)$ and $B^{c}(x, r)$ intersect each other and there exist $C_{1}, C_{2}>0$ such that $B^{c}(x, r) \subset B^{c}\left(a, C_{1} r\right) \subset$ $B^{c}\left(x, C_{2} r\right)$. Then, by the doubling property of $\mu^{c}$ we obtain the following chain of inequalities,

$$
\begin{aligned}
f_{B^{c}(x, r)}\left|u-u_{B^{c}(x, r)}\right| d \mu^{c} & \leq C \int_{B^{c}\left(a, C_{1} r\right)}\left|u-u_{B^{c}\left(a, C_{1} r\right)}\right| d \mu^{c} \\
& \leq C r\left(f_{6 K \lambda B^{c}\left(a, C_{1} r\right)} \bar{g}^{p} d \mu^{c}\right)^{1 / p} \\
& \leq C r\left(f_{6 K \lambda B^{c}\left(x, C_{2} r\right)} \bar{g}^{p} d \mu^{c}\right)^{1 / p}
\end{aligned}
$$


where in the second inequality we have applied the $p$-Poincaré inequality for balls in Case 2 centered at $a$.

Case 3: $1 / 4 \leq \lambda r d(x, c) \leq 4 \lambda L$. The proof of this case is similar to Case 3 of [20, Theorem 4.4].

Indeed, for $1 / 12 \leq \lambda r d(x, c) \leq 4 \lambda L$, we have that

$$
\begin{aligned}
f_{B^{c}(x, r)}\left|u-u_{B^{c}(x, r)}\right| d \mu^{c} & \leq 2 f_{B^{c}(x, r)}\left|u-u_{B^{c}(x, 48 \lambda L r)}\right| d \mu^{c} \\
& \leq C f_{B^{c}(x, 48 \lambda L r)}\left|u-u_{B^{c}(x, 48 \lambda L r)}\right| d \mu^{c} \\
& \leq C r\left(f_{288 K \lambda^{2} L B^{c}\left(x, C_{2} r\right)} \bar{g}^{p} d \mu^{c}\right)^{1 / p}
\end{aligned}
$$

where in the last inequality we have used the fact that $B^{c}(x, 8 r)$ satisfies the hypothesis of Case 2.

By combining the above three cases, we have proved the theorem.

The example [9, Example 4.12] gives a metric measure space endowed with a doubling measure, which is not meridian-like quasiconvexity, supporting an $\infty$-Poincaré inequality but whose flattened space fails to support an $\infty$-Poincaré inequality. Therefore, we cannot dispense of the hypothesis of meridian-like quasiconvexity in Theorem 4.1.

\section{The blow-up of a differentiability space does not need to be a PI space: An example}

It is an open question whether the blow-up (also known in the literature as tangent cone or tangent space) of a subset of positive measure of a differentiability space must be a PI space, that is, a metric space endowed with a doubling measure and a $p$-Poincaré inequality for some $p<\infty$. See, for example, [8]. Roughly speaking, the blow-up of a metric space at a point $x$ consists on "zooming into" $X$ close to $x$ and gives information about the infinitesimal behavior at the chosen point.

The following example is a modification of [11, Example 2] and shows that, at a particular point, this is not always the case.

ExAmple 2 . Let $Q=[0,1] \times[0,1] \subset \mathbb{R}^{2}$ be the unit square.

First, we divide $Q$ into nine equal squares of side-length $1 / 3$ and remove the central (open) one. We define the set $Q_{1}$ to be the union of the 8 remaining squares. Repeating this procedure on each of the 8 squares making up $Q_{1}$ we obtain the set $Q_{2}$, a union of $8^{2}$ squares, each of side-length $1 / 3^{2}$. Iterating this process, we get a sequence of sets $Q_{j}$ consisting of $8^{j}$ squares of side-length $1 / 3^{j}$. Because $Q_{j}$ has positive area for each $j$, we can define a probability measure $\mu_{j}$ concentrated on $Q_{j}$ obtained by renormalizing the 


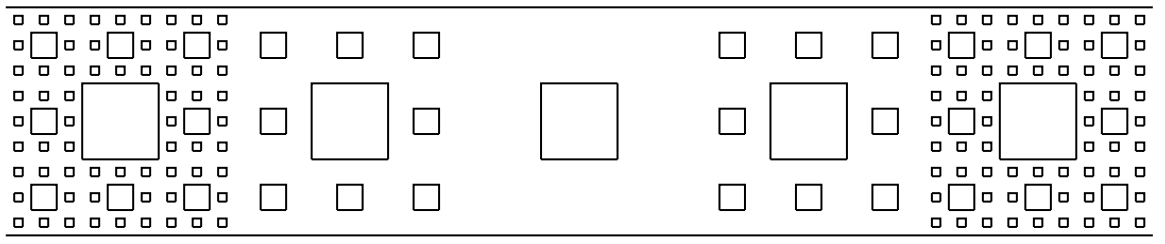

Figure 1. Metric measure pace $(X, d, \mu)$.

Lebesgue measure (restricted to $Q_{j}$ ) to have measure one. We now consider the following metric measure space (see Figure 1):

$$
X=\cdots \cup\left(Q_{3}+(-2,0)\right) \cup\left(Q_{2}+(-1,0)\right) \cup Q_{1} \cup\left(Q_{2}+(1,0)\right) \cup \cdots
$$

endowed with the measure

$$
\mu=\sum_{j=-1}^{\infty} \chi_{Q_{|j-1|}+(j, 0)} \cdot \mu_{j-1}+\sum_{j=1}^{\infty} \chi_{Q_{j}+(j-1,0)} \cdot \mu_{j},
$$

and with the Euclidean metric restricted to $X$. In the previous formula, $Q_{j}+(j-1,0)$ is the set obtained by translating $Q_{j}$ in the direction parallel to the $x$-axis by $j-1$ units and $\mu_{j}$ is the measure given by

$$
\mu_{j}=\left.(9 / 8)^{j} \mathscr{L}^{2}\right|_{Q_{j}+(j-1,0)} \text { for } j \in \mathbb{N},
$$

and

$$
\mu_{j}=\left.(9 / 8)^{|j|} \mathscr{L}^{2}\right|_{Q_{|j|}+(j+1,0)} \text { for } j \in \mathbb{Z}, j<0 .
$$

It can be directly verified that the measure $\mu$ is doubling on $X$.

As shown in [11], the space $(X, d, \mu)$ supports an $\infty$-Poincaré inequality but does not support any $p$-Poincaré inequality for finite $p$. This space, being a countable union of spaces with a Euclidean differentiable structure, is a metric differentiability space in the sense of Cheeger. Fix $a \in X$ and observe that $(X, d)$ is a radially star-like quasiconvex space. By [9, Theorem 3.4], the sphericalization $\left(\dot{X}, d_{a}, \mu_{a}\right)$ also supports an $\infty$-Poincaré inequality and a metric differentiable structure (given via the sphericalization of the metric differentiable structure of $(X, d, \mu))$. See [7] or [8] for the relevant definitions related to metric differentiable structures. However, $\left(\dot{X}, d_{a}, \mu_{a}\right)$ does not support a $p$-Poincaré inequality for any $p<\infty$. If that were the case, by Theorem 4.1, the flattening of $\left(\dot{X}, d_{a}, \mu_{a}\right)$, which is bi-Lipschitz equivalent to the original metric space $(X, d, \mu)$, would support a $q$-Poincaré inequality for some finite $q$. But, as mentioned above, this is not the case which yields a contradiction. Observe that $q$ might be different (but always finite) from $p$, being $p$ the exponent involved in the Poincaré inequality of the space $\left(\dot{X}, d_{a}, \mu_{a}\right)$. 
On the other hand, the blow-up of $\left(\dot{X}, d_{a}, \mu_{a}\right)$ at the point $x=\infty$ has a self-similar structure and coincides with $\left(\dot{X}, d_{a}, \mu_{a}\right)$, so it cannot support a $p$-Poincaré inequality for any $p<\infty$.

Acknowledgments. We are grateful to Prof. Nages Shanmugalingam for many valuable conversations concerning this paper. We would like to thank the referee for helpful comments and suggestions. We would also like to thank Prof. Andrea Schioppa for pointing out the precise open question posed in [8].

\section{REFERENCES}

[1] Z. Balogh and S. Buckley, Sphericalization and flattening, Conform. Geom. Dyn. 9 (2005), 76-101. MR 2179368

[2] A. Björn and J. Björn, Nonlinear potential theory on metric spaces, EMS Tracts in Mathematics, vol. 17, European Mathematical Society (EMS), Zürich, 2011. MR 2867756

[3] J. Björn and N. Shanmugalingam, Poincaré inequalities, uniform domains and extension properties for Newton-Sobolev functions in metric spaces, J. Math. Anal. Appl. 332 (2007), no. 1, 190-208. MR 2319654

[4] M. Bonk, J. Heinonen and P. Koskela, Uniformizing Gromov hyperbolic spaces, Astérisque 270 (2001), viii+99. MR 1829896

[5] M. Bonk and B. Kleiner, Rigidity for quasi-Möbius group actions, J. Differential Geom. 61 (2002), 81-106. MR 1949785

[6] S. Buckley, D. Herron and X. Xie, Metric inversions and quasihyperbolic geometry, Indiana Univ. Math. J. 57 (2008), no. 2, 837-890. MR 2414336

[7] J. Cheeger, Differentiability of Lipschitz functions on metric measure spaces, Geom. Funct. Anal. 9 (1999), 428-517. MR 1708448

[8] J. Cheeger, B. Kleiner and A. Schioppa, Infinitesimal structure of differentiability spaces, and metric differentiation, Anal. Geom. Metr. Spaces 4 (2016), 104-159. MR 3543676

[9] E. Durand-Cartagena and X. Li, Preservation of bounded geometry under sphericalization and flattening: Quasiconvexity and $\infty$-Poincaré inequality, Ann. Acad. Sci. Fenn. Math. 42 (2017), 1-22.

[10] E. Durand-Cartagena, N. Shanmugalingam and J. Jaramillo, Geometric characterizations of p-Poincaré inequalities in the metric setting, Publ. Mat. 60 (2016), no. 1, 81-111. MR 3447735

[11] E. Durand-Cartagena, N. Shanmugalingam and A. Williams, p-Poincaré inequality vs. $\infty$-Poincaré inequality; some counter-examples, Math. Z. 271 (2012), no. 1-2, 447-467. MR 2917152

[12] P. Hajłasz and K. Koskela, Sobolev met Poincaré, Mem. Amer. Math. Soc. 145 (2000), no. $688, \mathrm{x}+101$ pp. MR 1683160

[13] J. Heinonen, Lectures on analysis on metric spaces, Springer-Verlag, New York, 2001. MR 1800917

[14] J. Heinonen, T. Kilpeläinen and O. Martio, Nonlinear potential theory of degenerate elliptic equations, Oxford Univ. Press, New York, 1993. MR 1207810

[15] J. Heinonen and P. Koskela, Quasiconformal maps in metric spaces with controlled geometry, Acta Math. 181 (1998), 1-61. MR 1654771

[16] J. Heinonen, P. Koskela, N. Shanmugaligam and J. Tyson, Sobolev spaces on metric measure spaces: An approach based on upper gradients, New Mathematical Monographs, vol. 27, Cambridge University Press, 2015. MR 3363168 
[17] D. Herron, N. Shanmugalingam and X. Xie, Uniformity from Gromov hyperbolicity, Illinois J. Math. 52 (2008), no. 4, 1065-1109. MR 2595756

[18] S. Keith, Modulus and the Poincaré inequality on metric measure spaces, Math. Z. 245 (2003), no. 2, 255-292. MR 2013501

[19] R. Korte, Geometric implications of the Poincaré inequality, Results Math. 50 (2007), no. 1-2, 93-107. MR 2313133

[20] X. Li and N. Shanmugalingam, Preservation of bounded geometry under sphericalization and flattening, Indiana Univ. Math. J. 64 (2015), no. 5, 1303-1341. MR 3418443

[21] J. Mackay, J. Tyson and K. Wilkrick, Modulus and Poincaré inequalities on nonself-similar Sierpinski carpets, Geom. Funct. Anal. 23 (2013), no. 3, 985-1034. MR 3061778

Estibalitz Durand-Cartagena, Departamento de Matemática Aplicada, ETSi Industriales, Universidad Nacional de Educación a Distancia (UNED), Juan del Rosal 12, Ciudad Universitaria, 28040 Madrid, Spain

E-mail address: edurand@ind.uned.es

Xining Li, Department of Mathematics, Sun Yat-Sen University, Guangzhou, 510275, P. R. CHINA

E-mail address: lixining3@mail.sysu.edu.cn 